\title{
A Discrete Heterogeneous-Group Economic Growth Model with Endogenous Leisure Time
}

\author{
Wei-Bin Zhang \\ Ritsumeikan Asia Pacific University, 1-1 Jumonjibaru, Beppu-Shi, Oita-Ken 874-8577, Japan \\ Correspondence should be addressed to Wei-Bin Zhang, wbz1@apu.ac.jp
}

Received 5 July 2008; Revised 22 December 2008; Accepted 14 March 2009

Recommended by Weihong Huang

This paper proposes a one-sector multigroup growth model with endogenous labor supply in discrete time. Proposing an alternative approach to behavior of households, we examine the dynamics of wealth and income distribution in a competitive economy with capital accumulation as the main engine of economic growth. We show how human capital levels, preferences, and labor force of heterogeneous households determine the national economic growth, wealth, and income distribution and time allocation of the groups. By simulation we demonstrate, for instance, that in the three-group economy when the rich group's human capital is improved, all the groups will economically benefit, and the leisure times of all the groups are reduced but when any other group's human capital is improved, the group will economically benefit, the other two groups economically lose, and the leisure times of all the groups are increased.

Copyright ( $) 2009$ Wei-Bin Zhang. This is an open access article distributed under the Creative Commons Attribution License, which permits unrestricted use, distribution, and reproduction in any medium, provided the original work is properly cited.

\section{Introduction}

The purpose of this study is to study an economic growth model with heterogeneous households for providing insights into relations between economic growth and income and wealth distribution. In the economic growth literature, the Solow model is the starting point for almost all analyses of economic growth [1]. Nevertheless, the Solow model does not provide a mechanism of endogenous savings. Ramsey's 1928 paper on optimal growth theory has influenced modeling of consumers' behavior since the late 1960s [2]. This approach tends to be associated with higher dimensional dynamic systems. The approach often makes the analysis intractable even for a simple economic growth problem. In his original contribution to growth theory with capital accumulation, Diamond [3] used the overlapping generations structure as proposed by Samuelson [4] to examine the longterm dynamical efficiency of competitive production economies. The model has become a standard tool in macroeconomics to study economic dynamics in discrete time. These seminal papers were technically refined and generalized in different ways over years [57], and many other factors, such as human capital, population growth, and innovation, have 
been introduced into these analytical frameworks (e.g., [8-14]). The purpose of this study is to examine growth issues with endogenous time and heterogeneous groups. It should be remarked that multigroup growth models with endogenous savings can be found in literature of economic growth within the traditional approaches (see also, e.g., [15-18]). Our main deviation from these approaches is that we deal with the problem within a discrete framework with an alternative approach to behavior of consumers, as illustrated later. The paper is also an extension of Zhang's one-sector model with a homogenous population [19]. The paper is organized as follows. Section 2 defines the one-sector growth model with leisure time and heterogeneous groups. Section 3 analyzes the dynamic behavior of the two-group model. Section 4 examines impact of changes in some parameters on the wealth and income distribution. Section 5 simulates the 3-group model. Section 6 concludes the study.

\section{The Multigroup Growth Model in Discrete Time}

First, we develop a multigroup model in discrete time [20]. The economy has an infinite future. We represent the passage of time in a sequence of periods, numbered from zero and indexed by $t=0,1,2, \ldots$. Time 0 , being referred to the beginning of period 0 , represents the initial situation from which economy starts to grow. The end of period $t-1$ coincides with the beginning of period $t$; it can also be called period $t$. We assume that transactions are made in each period. The population is classified into groups, indexed by $j=1, \ldots, n$. Each type of consumers has a fixed number, denoted by $N_{j}$. As our model exhibits constant returns to scale, the dynamics (in terms of per capita) will not be affected if we allow the population to change at a constant growth rate over time. Let $K(t)$ denote the capital existing in period $t$ and $N(t)$ the flow of labor services used at time $t$ for production. Then $N(t)$ is given by

$$
N(t)=\sum_{j=1}^{n} h_{j} N_{j} T_{j}(t),
$$

where $h_{j}$ is the level of human capital of group $j, j=1, \ldots, n$, and $T_{j}(t)$ is the work time of a representative household of group $j$.

The production process is described by a neoclassical production function $F(t)=$ $F(K(t), N(t))[5,8]$. We assume that $F(K(t), N(t))$ is neoclassical. Introduce $f(k(t)) \equiv$ $F(k(t), 1)$, where $k(t) \equiv K(t) / N(t)$. The function $f$ has the following properties:

(i) $f(0)=0$;

(ii) $f$ is increasing, strictly concave on $R^{+}$and $C^{2}$ on $R^{++} ; f^{\prime}(k)>0$ and $f^{\prime \prime}(k)<0$;

(iii) $\lim _{k \rightarrow 0} f^{\prime}(k)=\infty$ and $\lim _{k \rightarrow+\infty} f^{\prime}(k)=0$.

Let $\delta_{k}$ denote the fixed rate of capital depreciation. Markets are competitive; thus labor and capital earn their marginal products, and firms earn zero profits. We assume that the output good serves as a medium of exchange and is taken as numeraire. The rate of interest, $r(t)$, and wage rate, $w(t)$, are determined by markets. Hence, for any individual firm $r(t)$ and $w(t)$ are given at each point of time. The production sector chooses the two variables $K(t)$ and $N(t)$ to maximize its profit. The marginal conditions are given by

$$
r(t)+\delta_{k}=f^{\prime}(k(t)), \quad w(t) \equiv f(k(t))-k(t) f^{\prime}(k(t)) .
$$

Let $k_{j}(t)$ denote per capita wealth of group $j$ in $t$. According to the definitions, we have $K(t)=$ $\sum_{j=1}^{n} k_{j}(t) N_{j}$. 
Divide the two sides of the above equation by $N(t)$ :

$$
k(t)=\sum_{j=1}^{n} k_{j}(t) n_{j}(t)
$$

where $n_{j}(t) \equiv N_{j} / N(t)$. From (2.1), we see that $r(t)$ and $w_{j}(t)$ are functions of $k_{j}(t)$ and $n_{j}(t)$.

Consumers make decisions on choice of consumption levels of services and commodities as well as on how much to save. In order to provide proper description of endogenous savings, we should know how individuals perceive the future. Different from the optimal growth theory in which utility defined over future consumption streams is used, we assume that we can find preference structure of consumers over leisure time, consumption, and saving at the current state. The preference over current and future consumption is reflected in the consumer's preference structure over leisure, consumption and saving. This study uses the approach to consumers' behavior proposed by Zhang. Theoretical and empirical implications and applications of the approach are examined in Zhang [21]. We now describe behavior of consumers. Group $j^{\prime}$ s per capita current income $y_{j}(t)$ from the interest payment $r(t) k_{j}(t)$ and the wage payment $w_{j}(t) T_{j}(t)$ is defined by

$$
y_{j}(t)=r(t) k_{j}(t)+w_{j}(t) T_{j}(t)
$$

The sum of money that consumers are using for consuming, saving, or transferring are not necessarily equal to the current income because consumers can sell wealth to pay, for instance, current consumption if the temporary income is not sufficient for purchasing goods and services. Retired people may not only live on the interest payment but also have to spend some of their wealth. The total value of wealth that consumer $j$ can sell to purchase goods and to save is equal to $k_{j}(t)$. Here, we do not allow borrowing for current consumption. We assume that selling and buying wealth can be conducted instantaneously without any transaction cost. This is evidently a strict consumption as it may take time to draw savings from bank or to sell one's properties. The per capita disposable income of consumer $j$ is defined as the sum of the current income and the wealth available for purchasing consumption goods and saving:

$$
\widehat{y}_{j}(t)=y_{j}(t)+k_{j}(t)=(1+r(t)) k_{j}(t)+w_{j}(t) T_{j}(t), \quad j=1, \ldots, n
$$

The disposable income is used for saving and consumption. At each point of time, a consumer would distribute the total available budget among savings, $s_{j}(t)$, and consumption of goods, $c_{j}(t)$. The budget constraint is given by

$$
c_{j}(t)+s_{j}(t)=\widehat{y}_{j}(t)
$$

Denote $T_{h j}(t)$ the leisure time at time $t$ and the (fixed) available time for work and leisure by $T_{0}$. The time constraint is expressed by $T_{j}(t)+T_{h j}(t)=T_{0}$. Substituting this function into the budget constraint yields

$$
w_{j}(t) T_{h j}(t)+c_{j}(t)+s_{j}(t)=\bar{y}_{j}(t) \equiv(1+r(t)) k_{j}(t)+w_{j}(t) T_{0}, \quad j=1, \ldots, n .
$$


At each point of time, consumers decide the three variables subject to the disposable income. We assume that utility level $U_{j}(t)$ is dependent on the leisure time, $T_{h j}(t)$, the consumption level of commodity, $c_{j}(t)$, and the savings, $s_{j}(t)$, as follows:

$$
U_{j}(t)=T_{h j}^{\sigma_{j}}(t) c_{j}^{\xi_{j}}(t) s_{j}^{\lambda_{j}}(t), \quad \sigma_{j}, \xi_{j}, \lambda_{j}>0, \sigma_{j}+\xi_{j}+\lambda_{j}=1, j=1, \ldots, n,
$$

where $\sigma_{j}, \xi_{j}$, and $\lambda_{j}$ are, respectively, group $j^{\prime}$ s propensities to consume and to hold wealth. Here, for simplicity, we specify the utility function with the Cobb-Douglas. It is important to examine dynamics with general utility functions. Maximizing $U_{j}$ subject to the budget constraints (2.7) yields

$$
w_{j}(t) T_{h j}(t)=\sigma_{j} \bar{y}_{j}(t), \quad c_{j}(t)=\xi_{j} \bar{y}_{j}(t), \quad s_{j}(t)=\lambda_{j} \bar{y}_{j}(t)
$$

Per capita wealth of group $j$ in period $t+1$ is equal to the savings made in period $t$, that is,

$$
k_{j}(t+1)=s_{j}\left(\bar{y}_{j}(t)\right), \quad j=1, \ldots, n \text {. }
$$

We will show that the above mappings control the motion of the system.

As output is either consumed or saved, the sum of net savings and consumption equals output, that is,

$$
C(t)+S(t)-K(t)+\delta_{k} K(t)=F(t)
$$

where $C(t)$ is the sum of consumption, $S(t)-K(t)+\delta_{k} K(t)$ is the sum of net savings of the groups, and $C(t)=\sum_{j} c_{j}(t) N_{j}$. It can be shown that (2.11) is redundant in the sense that it can be derived from the other equations in the system.

The dynamics consist of $n$-dimensional maps. In order to analyze properties of the dynamic system, it is necessary to express the dynamics in terms of $n$ variables. The following lemma, which is proved in Appendix A, shows that the dynamics is controlled by an $n$ dimensional maps system.

Lemma 2.1. The dynamics of the economic system is governed by the following $n$-dimensional difference equations:

$$
k_{j}(t+1)=\phi_{j}\left(k_{1}(t), \ldots, k_{n}(t)\right), \quad j=1, \ldots, n,
$$

where $\phi_{j}$ are differentiable functions of $k_{1}(t), \ldots, k_{n}(t)$. Moreover, all the other variables can be determined as functions of $k_{1}(t), \ldots, k_{n}(t)$ in any period by the following procedure: $k(t)$ by (A.9) $\rightarrow T_{j}(t)$ by $(\mathrm{A} .7) \rightarrow T_{h j}(t)=T_{0}-T_{j}(t) \rightarrow N(t)$ by $(2.1) \rightarrow r(t)$ and $w_{j}(t)$ by $(2.2) \rightarrow \bar{y}_{j}(t)$ by $(2.7) \rightarrow c_{j}(t)$ and $s_{j}(t)$ by $(2.9) \rightarrow K(t)=k(t) N(t) \rightarrow F(K(t), N(t)) \rightarrow f(t)=F(t) / N(t) \rightarrow$ $U_{j}(t)$ by $(2.8)$.

As it is difficult to find explicit conclusions about dynamic behavior of the system, in the remainder of this study we are concerned with a few special cases of the general model. 


\section{The Two-Group Model with the Cobb-Douglas Production Function}

This section is concerned with the case that there are two groups of labor force, and the production function takes on the Cobb-Douglas form by $f(k)=A k^{\alpha}$, where $0<\alpha<1$. As shown in (A.9) in Appendix A, $k$ is determined as a function of $k_{1}(t)$ and $k_{2}(t)$ by the following equation:

$$
\Lambda(k) \equiv k-\phi_{1}\left(k_{1}, k_{2}\right) k^{\beta}-\phi_{2}\left(k_{1}, k_{2}\right)=0,
$$

in which

$$
\begin{aligned}
& \phi_{1}\left(k_{1}, k_{2}\right) \equiv \sum_{j} k_{j} \bar{\sigma}_{j}>0, \quad \phi_{2}\left(k_{1}, k_{2}\right) \equiv \bar{A} K-\alpha \bar{A} \sum_{j} \bar{n}_{j} k_{j}>0, \\
& \bar{\sigma}_{j} \equiv \frac{\sigma_{j} N_{j} \delta \bar{A}}{A}>0, \quad \bar{n}_{j}=N_{j}\left(\xi_{j}+\lambda_{j}\right), \quad \bar{A} \equiv \frac{1}{\left(\sum_{j} h_{j} \bar{n}_{j}\right) \beta T_{0}}>0 .
\end{aligned}
$$

Equation (3.1) has a unique solution for given $k_{1}(t)$ and $k_{2}(t)$. The existence of at least one positive solution is guaranteed by $\Lambda(0)<0$ and $\Lambda(k) \rightarrow+\infty$ as $k \rightarrow+\infty$. Let the minimum positive solution of $\Lambda(k)=0$ be $K$. As

$$
\begin{aligned}
& \Lambda^{\prime}(K)=1-\beta \phi_{1} K^{-\alpha}=1-\phi_{1} K^{-\alpha}+\alpha \phi_{1} K^{-\alpha}=\frac{\phi_{2}}{K}+\alpha \phi_{1} K^{-\alpha}>0 \\
& \Lambda^{\prime \prime}(k)=\beta \alpha \phi_{1} k^{-1-\alpha}>0, \quad \forall k>0
\end{aligned}
$$

we conclude that $\Lambda^{\prime}(k)>0$ for any $k \geq K$. As $\Lambda(K)=0$, we see that it is impossible for any $k>K$ to satisfy $\Lambda(k)=0$. As $K$ is the minimum positive solution, the equation thus has a unique positive solution. We denote this solution by $k=\phi\left(k_{1}, k_{2}\right)$. According to (A.1) and with $f(k)=A k^{\alpha}$, we have

$$
\bar{y}_{j}(t)=\left(\alpha A k^{-\beta}+\delta\right) k_{j}+h_{j} \beta T_{0} A k^{\alpha}, \quad j=1,2
$$

Insert $s_{j}=\lambda_{j} \bar{y}_{j}$ and (3.4) into the difference equations (2.10):

$$
k_{j}(t+1)=\lambda_{j}\left[\left(\alpha A \phi^{-\beta}(t)+\delta\right) k_{j}(t)+h_{j} \beta T_{0} A \phi^{\alpha}(t)\right], \quad j=1,2 .
$$

The two difference equations contain only two variables, $k_{1}(t)$ and $k_{2}(t)$. With proper initial conditions $k_{1}(0)$ and $k_{2}(0)$, the two difference equations determine values of $k_{1}(t)$ and $k_{2}(t)$ in any period. According to Lemma 2.1, we can determine all the other variables in the system. Hence, it is sufficient to examine the dynamic properties of the two difference equations (3.5).

An equilibrium point of the system is given by

$$
h_{j} \beta T_{0} A \phi^{\alpha}=\left(\frac{1}{\lambda_{j}}-\delta-\alpha A \phi^{-\beta}\right) k_{j}
$$


At equilibrium we also have $\lambda_{j} \bar{y}_{j}=k_{j}$. From $\lambda_{j} \bar{y}_{j}=k_{j}$ and (3.4), we have

$$
k_{j}=\frac{h_{j} \beta T_{0} A k^{\alpha}}{\left(\bar{\lambda}_{j}-\alpha A k^{-\beta}\right)},
$$

where $\bar{\lambda}_{j} \equiv\left(\xi_{j}+\sigma_{j}\right) / \lambda_{j}+\delta_{k}>0$. To guarantee that $k_{j}$ is positive, we should require $\bar{\lambda}_{j}>\alpha A k^{-\beta}$. From (2.11), we have

$$
\sum_{j}\left(c_{j}+s_{j}\right) N_{j}=(f+\delta k) N
$$

Insert (2.9) and $\lambda_{j} \bar{y}_{j}=k_{j}$ into the above equation:

$$
\left(\tilde{\lambda}_{1}-A k^{-\beta}\right) N_{1} k_{1}+\left(\tilde{\lambda}_{2}-A k^{-\beta}\right) N_{2} k_{2}=0,
$$

where we use $k N=k_{1} N_{1}+k_{2} N_{2}$ and $\tilde{\lambda}_{j} \equiv \xi_{j} / \lambda_{j}+\delta_{k}>0$. Substitute (3.7) into the above equation:

$$
H(k) \equiv \frac{\left(k^{\beta} \tilde{\lambda}_{1}-A\right) h_{1} N_{1}}{\left(k^{\beta} \bar{\lambda}_{1}-\alpha A\right)}+\frac{\left(k^{\beta} \tilde{\lambda}_{2}-A\right) h_{2} N_{2}}{\left(k^{\beta} \bar{\lambda}_{2}-\alpha A\right)}=0 .
$$

As denominators are positive, for the equation to have solutions, $k^{\beta} \tilde{\lambda}_{1}-A$ and $k^{\beta} \tilde{\lambda}_{2}-A$ should have the opposite signs. For convenience of analysis, in the reminder of this we require $\tilde{\lambda}_{1}>\tilde{\lambda}_{2}$, that is, $\xi_{1} / \lambda_{1}>\xi_{2} / \lambda_{2}$. This requirement implies that group 1's "relative" propensity to save is higher than group 2's. Under this requirement, we have $k^{\beta} \tilde{\lambda}_{1}-A>0$ and $k^{\beta} \widetilde{\lambda}_{2}-A<0$. In Appendix B, we show that this equation has a unique (economically meaningful) solution. The following lemma is proved in Appendix B.

Lemma 3.1. The two-group economy has a unique equilibrium.

It should be noted that as discussed in Appendix B, the equilibrium may be either stable or unstable, depending on the parameter values. Now the impact of changes in some parameters is examined.

\section{Impact of Changes of Some Parameters in the Two-Group Model}

This section examines effects of changes in some parameters on the economic system. First, we study impact of change in group 1's human capital. Taking derivatives of (3.10) with respect to $h_{1}$,

$$
-\frac{d H}{d k} \frac{d k}{d h_{1}}=\frac{\left(k^{\beta} \tilde{\lambda}_{1}-A\right) N_{1}}{\left(k^{\beta} \bar{\lambda}_{1}-\alpha A\right)}>0,
$$

in which $d H / d k<0$ is given by (B.5). As $h_{1}$ is increased, the capital intensity, $k$, increases. As the productivity of group 1's labor force is increased, the average human capital tends to be 
increased. In the long term, the capital intensity in terms of the qualified labor input tends to be increased. From $r=\alpha A k^{-\beta}-\delta_{k}$ and $w_{j}=h_{j} \beta A k^{\alpha}$, we see that as group 1's level of human capital increases, the rate of interest falls, and the wage rates rise.

From (3.7), we have

$$
\begin{aligned}
& \frac{d k_{1}}{d h_{1}}=\frac{k_{1}}{h_{1}}+\left(\frac{\bar{\lambda}_{1}-A k^{-\beta}}{\bar{\lambda}_{1}-\alpha A k^{-\beta}}\right) \frac{\alpha k_{1}}{k} \frac{d k}{d h_{1}}, \\
& \frac{d k_{2}}{d h_{1}}=\left(\frac{\bar{\lambda}_{2}-A k^{-\beta}}{\bar{\lambda}_{2}-\alpha A k^{-\beta}}\right) \frac{\alpha k_{2}}{k} \frac{d k}{d h_{1}} .
\end{aligned}
$$

We see that if $\bar{\lambda}_{2}<(>) A k^{-\beta}$, then an increase in group 1's human capital reduces (increases) group 2's per capita wealth. As $\bar{\lambda}_{2}=\left(\xi_{2}+\sigma_{2}\right) / \lambda_{2}+\delta_{k}$, we see that $\bar{\lambda}_{2}>A k^{-\beta}$ tends to be satisfied if group 2 's propensity to save is relatively low. In the case that group 2 's propensity to save is relatively low, group 2's wealth per capita is increased when group 1's human capital rises. If $\bar{\lambda}_{1}>A k^{-\beta}$, then an increase in group 1 's human capital increases group 1 's per capita wealth. In the case of $\bar{\lambda}_{1}<A k^{-\beta}$, the impact on $k_{1}$ is ambiguous if no further requirement on the parameter values is added. We see that as group 1's human capital is improved, the impact on the capital intensity is certain but the effects on the levels of wealth per capita are ambiguous. From (B.6), we have

$$
\frac{d T_{h j}}{d h_{1}}=-\frac{\alpha A \beta k^{-\beta-1} T_{h j}}{\left(\bar{\lambda}_{j}-\alpha A k^{-\beta}\right)} \frac{d k}{d h_{1}}<0 .
$$

As group 1's level of human capital is increased, the leisure time of each group falls. As the human capital is improved, the capital intensity is increased. Consequently, the wage rate is increased. The value of work hour becomes higher for each group. Hence, the leisure time is reduced. By $\bar{y}_{j}=k_{j} / \lambda_{j}$ and $c_{j}=\xi_{j} k_{j} / \lambda_{j}$, we have

$$
\frac{d \bar{y}_{j}}{d h_{1}}=\frac{1}{\lambda_{j}} \frac{d k_{j}}{d h_{1}}, \quad \frac{d c_{j}}{d h_{1}}=\frac{\xi_{j}}{\lambda_{j}} \frac{d k_{j}}{d h_{1}}
$$

The effects on the disposable incomes and consumption levels have the same direction as that of the effect on the wealth per capita.

To study impact of preference change, we have to specify change pattern as $\sigma_{j}+\xi_{j}+$ $\lambda_{j}=1$. We are concerned with group 1's propensity to use leisure time, $\sigma_{1}$. We specify the preference change pattern by $d \sigma_{1}=-d \xi_{1}$ and $d \lambda_{1}=0$. That is, as the propensity to use leisure increases, the propensity to consume goods declines, and the propensity to save remains invariant. Taking derivatives of (3.10) with respect to $\sigma_{1}$,

$$
-\frac{d H}{d k} \frac{d k}{d \sigma_{1}}=-\frac{h_{1} N_{1}}{\left(k^{\beta} \bar{\lambda}_{1}-\alpha A\right) \lambda_{1}}<0 .
$$


As the propensity to consume leisure increases, $k$ decreases. As the household of group 1 values more leisure, the capital intensity is reduced. From (B.6), we have

$$
\begin{aligned}
& \frac{d T_{h 1}}{d \sigma_{1}}=\frac{T_{h 1}}{\sigma_{1}}-\frac{\alpha A \beta k^{-\beta-1} T_{h 1}}{\left(\bar{\lambda}_{1}-\alpha A k^{-\beta}\right)} \frac{d k}{d \sigma_{1}}>0, \\
& \frac{d T_{h 2}}{d \sigma_{1}}=-\frac{\alpha A \beta k^{-\beta-1} T_{h 2}}{\left(\bar{\lambda}_{2}-\alpha A k^{-\beta}\right)} \frac{d k}{d \sigma_{1}}>0 .
\end{aligned}
$$

As group 1's propensity to use leisure increases, the leisure time of each group rises. From $r=\alpha A k^{-\beta}-\delta_{k}$ and $w_{j}=h_{j} \beta A k^{\alpha}$, we see that as group 1's propensity to use leisure time increases, the rate of interest rises, and the wage rates fall. From (3.6), we have

$$
\frac{d k_{j}}{d \sigma_{1}}=\left(\frac{\bar{\lambda}_{j}-A k^{-\beta}}{\bar{\lambda}_{j}-\alpha A k^{-\beta}}\right) \frac{\alpha k_{j}}{k} \frac{d k}{d \sigma_{1}} .
$$

We see that if $\bar{\lambda}_{j}<(>) A k^{-\beta}$, then an increase in group 1's propensity to enjoy leisure reduces (increases) group $j$ 's per capita wealth. By $\bar{y}_{j}=k_{j} / \lambda_{j}$ and $c_{j}=\xi_{j} k_{j} / \lambda_{j}$, we have

$$
\frac{d \bar{y}_{j}}{d \sigma_{1}}=\frac{1}{\lambda_{j}} \frac{d k_{j}}{d \sigma_{1}}, \quad \frac{d c_{1}}{d \sigma_{1}}=\frac{\xi_{1}}{\lambda_{1}} \frac{d k_{1}}{d \sigma_{1}}-\frac{k_{1}}{\lambda_{1}}, \quad \frac{d c_{2}}{d \sigma_{1}}=\frac{\xi_{21}}{\lambda_{2}} \frac{d k_{2}}{d \sigma_{1}}
$$

\section{Simulating the 3-Group Model}

This section simulates the model when the economy consists of three different groups. For illustration, we specify $\alpha=1 / 3$. That is, the production function takes on the CobbDouglas form, $f(k)=A k^{1 / 3}$. The choice does not seem to be unrealistic. For instance, some empirical studies on the US economy demonstrate that the value of the parameter, $\alpha$, in the Cobb-Douglas production is approximately equal to 0.3 (e.g., [22]). As shown in (A.9) in Appendix A, $k$ is determined as a function of $k_{j}(t), j=1,2,3$ by the following equation:

$$
\Lambda(k) \equiv k-\phi_{1} k^{2 / 3}-\phi_{2}=0,
$$

in which

$$
\begin{gathered}
\phi_{1} \equiv \sum_{j=1}^{3} k_{j} \bar{\sigma}_{j}>0, \quad \phi_{2} \equiv \bar{A} K-\alpha \bar{A} \sum_{j=1}^{3} \bar{n}_{j} k_{j}>0, \\
\bar{\sigma}_{j} \equiv \frac{\sigma_{j} N_{j} \delta \bar{A}}{A}>0, \quad \bar{n}_{j}=N_{j}\left(\xi_{j}+\lambda_{j}\right), \quad \bar{A} \equiv \frac{1}{\left(\sum_{j=1}^{5} h_{j} \bar{n}_{j}\right) \beta T_{0}}>0 .
\end{gathered}
$$


Equation (5.1) has a unique solution for given $k_{j}(t)$. The solution is explicitly given by

$$
k(t)=\phi\left(\left\{k_{j}(t)\right\}\right)=\left[\frac{\phi_{1}}{3}+\frac{2^{1 / 3} \phi_{1}^{2}}{3\left(2 \phi_{1}^{3}+27 \phi_{2}+3 \phi_{0} \sqrt{3 \phi_{2}}\right)^{1 / 3}}+\frac{\left(2 \phi_{1}^{3}+27 \phi_{2}+3 \phi_{0} \sqrt{3 \phi_{2}}\right)^{1 / 3}}{3 \sqrt{2}}\right]^{3},
$$

where

$$
\phi_{0}\left(\left\{k_{j}(t)\right\}\right) \equiv\left[4 \phi_{1}^{2}\left\{k_{j}(t)\right\}+27 \phi_{2}\left\{k_{j}(t)\right\}\right]^{1 / 2} .
$$

According to (A.1) and with $f(k)=A k^{\alpha}$, we have

$$
\bar{y}_{j}(t)=\left(\frac{A k^{-2 / 3}}{3}+\delta\right) k_{j}+\frac{2 h_{j} T_{0} A k^{1 / 3}}{3}, \quad j=1,2,3
$$

Insert $s_{j}=\lambda_{j} \bar{y}_{j}$ and (5.5) into the difference equations: (2.10)

$$
k_{j}(t+1)=\lambda_{j}\left[\left(\frac{A \phi^{-2 / 3}\left(\left\{k_{j}\right\}\right)}{3}+\delta\right) k_{j}+\frac{2 h_{j} T_{0} A \phi^{1 / 3}\left(\left\{k_{j}\right\}\right)}{3}\right], \quad j=1,2,3 .
$$

The three difference equations contain three variables, $\left\{k_{j}(t)\right\}$. With proper initial conditions $\left\{k_{j}(0)\right\}$, these difference equations determine values of $\left\{k_{j}(t)\right\}$ in any period. According to Lemma 2.1, we can determine all the other variables in the system. Hence, it is sufficient to examine the dynamic properties of the difference equations (5.3) as with the two-group case in the previous sections.

At equilibrium we have $\lambda_{j} \bar{y}_{j}=k_{j}$. From $\lambda_{j} \bar{y}_{j}=k_{j}$ and (5.5), we solve $\left\{k_{j}\right\}$ as in (3.7). Similar to (3.10), the equilibrium value of $k$ is given by

$$
H(k) \equiv \sum_{j=1}^{3} \frac{\left(k^{2 / 3} \tilde{\lambda}_{j}-A\right) h_{j} N_{j}}{\left(k^{2 / 3} \bar{\lambda}_{j}-A / 3\right)}=0
$$

Similar to the requirements (B.1)-(B.3), we should require $k$ to satisfy certain conditions for the equilibrium solution to be meaningful.

Rather than further examining these conditions, we simulate the model. To simulate the model, we specify the groups' human capital and preferences as follows:

$$
\begin{array}{ll}
\left(\begin{array}{l}
h_{1} \\
h_{2} \\
h_{3}
\end{array}\right)=\left(\begin{array}{c}
13 \\
4 \\
1
\end{array}\right), \quad\left(\begin{array}{l}
\lambda_{1} \\
\lambda_{2} \\
\lambda_{3}
\end{array}\right)=\left(\begin{array}{l}
0.65 \\
0.55 \\
0.25
\end{array}\right), \\
\left(\begin{array}{l}
\sigma_{1} \\
\sigma_{2} \\
\sigma_{3}
\end{array}\right)=\left(\begin{array}{c}
0.29 \\
0.3 \\
0.45
\end{array}\right), & \left(\begin{array}{l}
N_{1} \\
N_{2} \\
N_{3}
\end{array}\right)=\left(\begin{array}{c}
3 \\
82 \\
15
\end{array}\right) .
\end{array}
$$


Group 1 is called the rich class-with the highest level of human capital and the highest propensity to own wealth. The population share of the rich in the total population is only 3 percent. Group 2 is termed as the working class. Its population share is 82 percent. Group 3 is the poor class. The human capital level of this group is low, and its propensity to save is low. The share of the population is 15 percent. We specify the rest three parameters as follows

$$
A=3.3, \quad \delta_{k}=0.06, \quad T_{0}=24 \text {. }
$$

The simulation demonstrates a unique equilibrium value of $k=34.08$. The equilibrium values of the other variables are as follows:

$$
\begin{gathered}
K=86975.5, \quad F=27305.6, \quad r=0.045, \\
\left(\begin{array}{l}
k_{1} \\
k_{2} \\
k_{3}
\end{array}\right)=\left(\begin{array}{c}
4506.55 \\
885.21 \\
57.92
\end{array}\right), \quad\left(\begin{array}{l}
T_{h 1} \\
T_{h 2} \\
T_{h 3}
\end{array}\right)=\left(\begin{array}{l}
21.68 \\
16.92 \\
14.62
\end{array}\right), \\
\left(\begin{array}{l}
c_{1} \\
c_{2} \\
c_{3}
\end{array}\right)=\left(\begin{array}{c}
415.99 \\
241.42 \\
69.51
\end{array}\right), \quad\left(\begin{array}{c}
w_{1} \\
w_{2} \\
w_{3}
\end{array}\right)=\left(\begin{array}{c}
92.73 \\
28.53 \\
7.13
\end{array}\right) .
\end{gathered}
$$

The wealth per capita of the rich group is 5 times as that of the working class, and the wealth per capita of the working class is 15 times as that of the poor class. The rich class's population is only 3 percent but its shares of income and wealth are, respectively, 5.65 and 15.54 percent; the middle class's share of population is 82 percent, and its shares of income and wealth are, respectively, 89.6 and 83.5 percent; the poor class's share of population is 15 percent, and its shares of income and wealth are, respectively, 4.72 and 0.01 . The rich class enjoys the highest leisure time. The poor class has the least leisure time. The rich class consumes much more than the poor class. Due to the human capital differences, the three groups have different wage rates. The three eigenvalues are given by

$$
\rho_{1,2}=22998.6 \pm 277585 i, \quad \rho_{3}=0.69 .
$$

The steady state is unstable. Further simulation results demonstrate that the system may be either stable or unstable, depending on the parameter values. Since the stability conditions are difficult to interpret, we do not further examine them.

As the dynamic system has a unique equilibrium, we can examine impact of changes in the parameters. First, we examine impact of change in human capital. We fix the parameter values as in (5.8) and (5.9) except one parameter $h_{1}$. We increase the rich class's level of human capital from 13 to 14 . We calculate the new equilibrium values as

$$
\begin{aligned}
\Delta k=31, \quad & \Delta K=1228.27, \quad \Delta F=218.72, \quad \Delta r=-0.001, \\
\Delta h_{1}=1: & \left(\begin{array}{l}
\Delta k_{1} \\
\Delta k_{2} \\
\Delta k_{3}
\end{array}\right)=\left(\begin{array}{c}
355.14 \\
1.96 \\
0.16
\end{array}\right),\left(\begin{array}{l}
\Delta T_{h 1} \\
\Delta T_{h 2} \\
\Delta T_{h 3}
\end{array}\right)=\left(\begin{array}{l}
-0.03 \\
-0.01 \\
-0.01
\end{array}\right), \\
& \left(\begin{array}{l}
\Delta c_{1} \\
\Delta c_{2} \\
\Delta c_{3}
\end{array}\right)=\left(\begin{array}{c}
32.78 \\
1.53 \\
0.20
\end{array}\right),\left(\begin{array}{l}
\Delta w_{1} \\
\Delta w_{2} \\
\Delta w_{3}
\end{array}\right)=\left(\begin{array}{l}
7.44 \\
0.09 \\
0.02
\end{array}\right) .
\end{aligned}
$$


In (5.12), we denote the difference between equilibrium values of the variables at the new equilibrium point and old one by $\Delta$. From (5.12), we see that as the rich class's human capital is increased, the total output, the total wealth and per capita wealth of all the groups, the wage rates, and consumption are all increased but the leisure times of all the groups are slightly reduced. Hence, every group and the society as a whole benefit from human capital improvement of the rich class. It should be remarked that the poor class benefits only slightly from the change. This implies that as the rich class improves its human capital, the poor class's relative "social status" might become lower. The wealth and income gaps are enlarged among the classes.

We increase levels of human capital of the other two classes as follows:

$$
\begin{aligned}
& \Delta k=-0.52, \quad \Delta K=17767.1, \quad \Delta F=5914.91, \quad \Delta r=0.001 \text {, } \\
& \Delta h_{2}=1:\left(\begin{array}{l}
\Delta k_{1} \\
\Delta k_{2} \\
\Delta k_{3}
\end{array}\right)=\left(\begin{array}{c}
-13.15 \\
217.20 \\
-0.27
\end{array}\right),\left(\begin{array}{l}
\Delta T_{h 1} \\
\Delta T_{h 2} \\
\Delta T_{h 3}
\end{array}\right)=\left(\begin{array}{c}
0.05 \\
0.02 \\
0.01
\end{array}\right), \\
& \left(\begin{array}{l}
\Delta c_{1} \\
\Delta c_{2} \\
\Delta c_{3}
\end{array}\right)=\left(\begin{array}{c}
-1.22 \\
59.24 \\
-0.33
\end{array}\right),\left(\begin{array}{l}
\Delta w_{1} \\
\Delta w_{2} \\
\Delta w_{3}
\end{array}\right)=\left(\begin{array}{c}
-0.47 \\
6.95 \\
-0.04
\end{array}\right) \\
& \Delta k=-2.77, \quad \Delta K=-4.96, \quad \Delta F=1585.57, \quad \Delta r=0.006 \text {, } \\
& h_{3}=2:\left(\begin{array}{l}
\Delta k_{1} \\
\Delta k_{2} \\
\Delta k_{3}
\end{array}\right)=\left(\begin{array}{l}
-70.89 \\
-17.84 \\
111.36
\end{array}\right),\left(\begin{array}{l}
\Delta T_{h 1} \\
\Delta T_{h 2} \\
\Delta T_{h 3}
\end{array}\right)=\left(\begin{array}{c}
0.27 \\
0.13 \\
0.03
\end{array}\right) \text {, } \\
& \left(\begin{array}{l}
\Delta c_{1} \\
\Delta c_{2} \\
\Delta c_{3}
\end{array}\right)=\left(\begin{array}{c}
-6.54 \\
-4.87 \\
133.63
\end{array}\right),\left(\begin{array}{l}
\Delta w_{1} \\
\Delta w_{2} \\
\Delta w_{3}
\end{array}\right)=\left(\begin{array}{c}
-2.58 \\
-0.80 \\
13.67
\end{array}\right) \text {. }
\end{aligned}
$$

We see that as the working class's human capital is improved, the levels of per capita wealth and consumption and wage rates of the working class are increased and the levels of per capita wealth and consumption and wage rates of the other two classes fall. As the poor class improves its human capital, the class's living conditions and wealth are improved but the other two classes do not benefit, except that they have more leisure time. When the rich class increases its level of human capital, the rate of interest decreases but when any of the other two classes increases its level of human capital, the rate of interest increases. Here, we see that changes in human capital of different groups have different implications for different groups.

We now examine the impact of technological parameter on the equilibrium values of the dynamic system. We list up the effects on the variables as follows:

$$
\begin{array}{r}
\Delta k=11.40, \quad \Delta K=29093.4, \quad \Delta F=9133.75, \quad \Delta r \approx 0, \\
\Delta A=0.7:\left(\begin{array}{c}
\Delta k_{1} \\
\Delta k_{2} \\
\Delta k_{3}
\end{array}\right)=\left(\begin{array}{c}
1507.45 \\
296.10 \\
19.38
\end{array}\right),\left(\begin{array}{l}
\Delta T_{h 1} \\
\Delta T_{h 2} \\
\Delta T_{h 3}
\end{array}\right) \approx\left(\begin{array}{l}
0 \\
0 \\
0
\end{array}\right), \\
\left(\begin{array}{c}
\Delta c_{1} \\
\Delta c_{2} \\
\Delta c_{3}
\end{array}\right)=\left(\begin{array}{c}
139.15 \\
80.76 \\
23.25
\end{array}\right),\left(\begin{array}{l}
\Delta w_{1} \\
\Delta w_{2} \\
\Delta w_{3}
\end{array}\right)=\left(\begin{array}{c}
31.02 \\
9.54 \\
2.39
\end{array}\right) .
\end{array}
$$


As the technology is improved, the levels of per capita wealth, consumption, and wage rate of all the classes are increased. The rate of interest is slightly affected by the technological change. We see that the rich class benefits most from the general improvement. Although the technological change benefits all the classes, the income and wealth gaps among the classes are enlarged.

We also examine effects of change in the preferences. We increase the propensity to save by $\Delta \lambda_{j}$ and reduce the propensities to consume and use leisure time by $-\Delta \lambda_{j} / 2$, respectively. The simulation results show that as any class's propensity to save is increased, the rate of interest declines; the capital intensity of production increases; the total capital stocks, the total output, and the wage rates of all the classes are increased. When any class increases its propensity to save, its leisure time is increased but the two other classes' leisure times are reduced. We also simulated the effects of change in each group's population. As any group's labor force increases, the total output level is increased, and the rate of interest is slightly affected. When the rich class increases its population, the levels of per capita wealth, the wage rates, and the consumption levels of all the classes are increased, and the leisure times of all the classes are reduced. When the working class or poor class increases its population, the wage rates, and the levels of per capita wealth and of consumption of all the classes are reduced and the leisure times are increased. As the simulations are straightforward, we will not provide the results here.

\section{Conclusions}

We proposed a one-sector growth multigroup model with endogenous labor supply to provide some insights into dynamics of wealth and income distribution in a competitive economy with capital accumulation as the main engine of economic growth. This study treats capital accumulation as the main engine of economic growth. It is known that almost all the contemporary growth models with microeconomic foundation are based on Ramsey's 1928 paper. As the Ramsey [2] model provides a rational mechanism of household behavior, it is reasonable to expect that the homogenous population Ramsey model has been extended to economies with heterogeneous households over years. It has become clear that the Ramsey growth model with heterogeneous households tends to result in dynamically intractable problems. A typical model of the Ramsey approach is reflected in a model of heterogeneous households by Becker [15]. The model forges a link between income distribution, wealth distribution, and economic growth. The Becker model demonstrates that if an agent's lifetime utility function over an infinite horizon is represented by a stationary, additive, discounted function with a constant pure rate of time preference, then the income distribution is shown in the long-run steady state to be determined by the lowest discount rate. The household (e.g., a single household) with the lowest rate of discount owns all the capital and earns a wage income; all other households (e.g., other twenty millions households) receive a wage income and have no wealth. Different from the standard Ramsey model, the model in this paper shows nondegenerate long-run distribution among the heterogeneous households. This paper also demonstrates the importance of introducing heterogeneous households into the growth theory. By simulation we demonstrate, for instance, that in the three-group economy when the rich group's human capital is improved, all the groups will economically benefit, and the leisure times of all the groups are reduced, but when any other group's human capital is improved, the group will economically benefit, the other two groups economically lose, and the leisure times of all the groups are increased. We show that the same change in different groups may have different implications for the national economy. 
An immediate and important extension of the model is to make technological change, human capital, preferences as well as the labor force (which all have been fixed in this study) as endogenous variables. There is huge amount of literature about endogenous technological change, human capital accumulation, preference change, and population in economics. It would be fruitful to examine different issues of economic growth and development within the framework proposed in this study.

\section{Appendices}

\section{A. Proving Lemma 2.1}

We now prove Lemma 2.1 in Section 2. From the definition of $\bar{y}_{j}(t)$ in $(2.7)$ and (2.1), we have

$$
\bar{y}_{j}(t)=f_{\delta}(k) k_{j}+h_{j} f_{0}(k)
$$

in which

$$
f_{\delta}(k) \equiv f^{\prime}(k)+\delta>0, \quad f_{0}(k) \equiv\left[f(k)-k f^{\prime}(k)\right] T_{0}>0, \quad \forall k>0 .
$$

From $w_{j} T_{h j}=\sigma_{j} \bar{y}_{j}$, we have

$$
\frac{T_{h 1}}{T_{h j}}=\frac{\bar{\sigma}_{j} \bar{y}_{1}}{\bar{y}_{j}}, \quad j=2, \ldots, n,
$$

where we use $w_{1} / w_{j}=h_{1} / h_{j}$ and

$$
\bar{\sigma}_{j} \equiv \frac{h_{j} \sigma_{1}}{h_{1} \sigma_{j}}, \quad j=1, \ldots, n
$$

From $T_{h j}=T_{0}-T_{j}$, (A.1), and (A.3), we solve

$$
T_{j}=\frac{\left[\left(\bar{\sigma}_{j} k_{1}-k_{j}\right) f_{\delta}+\bar{h}_{j} f_{0}\right] T_{0}+\left(k_{j} f_{\delta}+h_{j} f_{0}\right) T_{1}}{\left(k_{1} f_{\mathcal{\delta}}+h_{1} f_{0}\right) \bar{\sigma}_{j}}, \quad j=2, \ldots, n,
$$

where $\bar{h}_{j} \equiv \bar{\sigma}_{j} h_{1}-h_{j}$. From (2.1) and (2.3), we have

$$
\sum_{j=1}^{n} h_{j} N_{j} T_{j}(t)=\frac{K(t)}{k(t)} .
$$


Solve (A.5) and (A.6) with $T_{j}(t)$ as variables

$$
\begin{aligned}
& T_{1}(t)=\Omega_{1}(k) \equiv\left[\frac{K}{k}-\sum_{j=2}^{n} \frac{\left[\left(\bar{\sigma}_{j} k_{1}-k_{j}\right) f_{\delta}+\bar{h}_{j} f_{0}\right] h_{j} N_{j} T_{0}}{\left(k_{1} f_{\delta}+h_{1} f_{0}\right) \bar{\sigma}_{j}}\right]\left(h_{1} N_{1}+\sum_{j=2}^{n} \frac{\left(k_{j} f_{\delta}+h_{j} f_{0}\right) h_{j} N_{j}}{\left(k_{1} f_{\delta}+h_{1} f_{0}\right) \bar{\sigma}_{j}}\right)^{-1}, \\
& T_{j}(t)=\Omega_{j}\left(k, k_{1}, \ldots, k_{n}\right) \equiv \frac{\left[\left(\bar{\sigma}_{j} k_{1}-k_{j}\right) f_{\delta}+\bar{h}_{j} f_{0}\right] T_{0}+\left(k_{j} f_{\delta}+h_{j} f_{0}\right) \Omega_{1}}{\left(k_{1} f_{\delta}+h_{1} f_{0}\right) \bar{\sigma}_{j}}, \quad j=2, \ldots, n .
\end{aligned}
$$

As $K, f_{0}, f_{\delta}$, and $f$ are all functions of $k$ and $\left\{k_{j}\right\}$, the above equations show that the work times of all the groups are uniquely determined as functions of $k$ and $\left\{k_{j}\right\}$.

From (2.11), we have

$$
\sum_{j}\left(c_{j}+s_{j}\right) N_{j}=F+\delta K=(f+\delta k) N
$$

Insert (A.1), (2.1), and $c_{j}$ and $s_{j}$ in (2.11) into the above equation:

$$
\Lambda\left(k ; k_{1}, k_{2}\right) \equiv\left(\frac{f(k)}{k}+\delta\right) K-\sum_{j} N_{j}\left(\xi_{j}+\lambda_{j}\right)\left(k_{j} f_{\delta}(k)+h_{j} f_{0}(k)\right)=0,
$$

where we use $N=K / k$. We now show that for any given $k_{j} \geq 0$ for all $j, \Lambda(k)=0$ has at least one positive solution. Note that $K>0$ (when at least one $k_{j}>0$ ) is a function of $\left\{k_{j}\right\}$. According to the definitions of $f_{0}$ and $f_{\delta}$, and the properties of $f$, it is straightforward to check the following properties of $\Lambda(k)$ :

$$
\Lambda(k) \longrightarrow+\infty \text { as } k \longrightarrow 0, \quad \Lambda(k) \longrightarrow-\infty \text { as } k \longrightarrow+\infty,
$$

where we use

$$
\begin{gathered}
\frac{f}{k} \longrightarrow+\infty \quad \text { as } k \longrightarrow 0, \quad \frac{f}{k} \longrightarrow 0 \quad \text { as } k \longrightarrow+\infty, \\
f^{\prime}-\frac{f}{k}<0, \quad f^{\prime}>0, \quad f^{\prime \prime}<0, \quad \forall k>0 .
\end{gathered}
$$

We see that $\Lambda(k)=0$ has at least one positive solution. The solution can be expressed as a function of $\left\{k_{j}\right\}$. Take derivative of $\Lambda(k)$ with respect to $k$ :

$$
\frac{d \Lambda}{d k}=\left(f^{\prime}-\frac{f}{k}\right) \frac{K}{k}-\sum_{j} N_{j}\left(\xi_{j}+\lambda_{j}\right)\left(k_{j}-h_{j} T_{0} k\right) f^{\prime \prime}
$$

As the sign of $d \Lambda / d k$ is ambiguous, we are not sure about the uniqueness of solution. As $k(t)$ is a function of $\left\{k_{j}\right\}$ at any point of time, from (A.1) we see that $\bar{y}_{j}(t)$ are functions of $\left\{k_{j}\right\}$. 
Hence, from (2.10), (A.1), and $s_{j}=\lambda_{j} \bar{y}_{j}$, we get

$$
k_{j}(t+1)=\phi_{j}\left(k_{1}(t), \ldots, k_{n}(t)\right) \equiv \lambda_{j}\left\lfloor f_{\delta}(k) k_{j}+h_{j} f_{0}(k)\right\rfloor, \quad j=1, \ldots, n .
$$

\section{B. Proving Lemma 3.1}

We now show that (3.10) has a unique solution. From (3.7), we see that it is necessary for $k$ to satisfy

$$
k>\max _{j}\left\{\left(\frac{\alpha A}{\bar{\lambda}_{j}}\right)^{1 / \beta}\right\}
$$

For (3.10) to positive solution, it is necessary to ask for $\tilde{\lambda}_{1}-A k^{-\beta}$ and $\tilde{\lambda}_{2}-A k^{-\beta}$ to have the opposite sign if $\tilde{\lambda}_{1} \neq \tilde{\lambda}_{2}$. If $\tilde{\lambda}_{1}=\tilde{\lambda}_{2}$, then $k=\left(A / \tilde{\lambda}_{2}\right)^{1 / \beta}$, which also satisfies (B.1). For convenience of analysis, let $\tilde{\lambda}_{1}>\tilde{\lambda}_{2}$, that is, $\xi_{1} / \lambda_{1}>\xi_{2} / \lambda_{2}$. From (3.10) and (B.1), we should require

$$
\left(\frac{A}{\tilde{\jmath}_{2}}\right)^{1 / \beta}>k>\left(\frac{A}{\tilde{\lambda}_{1}}\right)^{1 / \beta}
$$

As $\tilde{\lambda}_{1}>\tilde{\lambda}_{2}$, we always have $A / \tilde{\lambda}_{j}>\alpha A / \bar{\lambda}_{j}$. Hence for the requirements (B.1) and (B.2) to satisfy, we should require

$$
\left(\frac{A}{\tilde{\jmath}_{2}}\right)^{1 / \beta}>k>\lambda^{*} \equiv \max \left\{\left(\frac{A}{\tilde{\jmath}_{1}}\right)^{1 / \beta},\left(\frac{\alpha A}{\bar{\lambda}_{2}}\right)^{1 / \beta}\right\} .
$$

It is straightforward to check the following properties of $H(k)$ :

$$
\begin{gathered}
H\left(\left(\frac{A}{\tilde{\lambda}_{2}}\right)^{1 / \beta}\right)=\frac{\left(\tilde{\lambda}_{1} / \tilde{\lambda}_{2}-1\right) h_{1} N_{1}}{\left(\bar{\lambda}_{1} / \tilde{\lambda}_{2}-\alpha\right)}>0, \\
H\left(\lambda^{*}\right)=\frac{\left(\tilde{\lambda}_{2} / \tilde{\lambda}_{1}-1\right) h_{2} N_{2}}{\left(\bar{\lambda}_{2} / \tilde{\lambda}_{1}-\alpha\right)}<0, \quad \text { if } \lambda^{*}=\left(\frac{A}{\tilde{\lambda}_{1}}\right)^{1 / \beta}, \\
H\left(\lambda^{*}\right)<0, \quad \text { if } \lambda^{*}=\left(\frac{\alpha A}{\bar{\lambda}_{2}}\right)^{1 / \beta} .
\end{gathered}
$$

Accordingly, $H(k)=0$ has at least positive solution which satisfies (B.3). Take derivatives of $H(k)$ with respect to $k$ :

$$
\frac{d H}{d k}=\left[\frac{\left(\bar{\lambda}_{1}-\alpha \tilde{\lambda}_{1}\right)}{\left(k^{\beta} \bar{\lambda}_{1}-\alpha A\right)^{2}} h_{1} N_{1}+\frac{\left(\bar{\lambda}_{2}-\alpha \tilde{\lambda}_{2}\right)}{\left(k^{\beta} \bar{\lambda}_{2}-\alpha A\right)^{2}} h_{2} N_{2}\right] A \beta k^{-\alpha}<0 .
$$

From (B.4) and (B.5), we conclude that $H(k)=0$ has a unique solution. 
From $T_{h j}=\sigma_{j} \bar{y}_{j} / w_{j}, \bar{y}_{j}=k_{j} / \lambda_{j}$, and $w_{j}=\beta A k^{\alpha}$, we have

$$
T_{h j}=\frac{\sigma_{j} k_{j}}{\beta A h_{j} k^{\alpha} \lambda_{j}}=\frac{\sigma_{j} T_{0}}{\lambda_{j}} \frac{1}{\left(\bar{\lambda}_{j}-\alpha A k^{-\beta}\right)} .
$$

We see that under (B.3), we have $0<T_{h j}<T_{0}$. According to Lemma 2.1, we can determine all the other variables. Hence, we proved that the system has a unique equilibrium.

We now determine stability of the unique equilibrium. The Jacobian matrix at equilibrium is given by

$$
J=\left[\begin{array}{ll}
a_{11} & a_{12} \\
a_{21} & a_{22}
\end{array}\right]
$$

where

$$
\begin{aligned}
& a_{11}=\left(\frac{\alpha A}{k^{\beta}}+\delta\right) \lambda_{1}+\left(h_{1} T_{0}-\frac{k_{1}}{k}\right) \frac{\lambda_{1} \alpha \beta A}{k^{\beta}} \frac{\partial \phi}{\partial k_{1}}, \quad a_{12}=\left(h_{1} T_{0}-\frac{k_{1}}{k}\right) \frac{\lambda_{1} \alpha \beta A}{k^{\beta}} \frac{\partial \phi}{\partial k_{2}}, \\
& a_{21}=\left(h_{2} T_{0}-\frac{k_{2}}{k}\right) \frac{\lambda_{2} \alpha \beta A}{k^{\beta}} \frac{\partial \phi}{\partial k_{1}}, \quad a_{22}=\left(\frac{\alpha A}{k^{\beta}}+\delta\right) \lambda_{2}+\left(h_{2} T_{0}-\frac{k_{2}}{k}\right) \frac{\lambda_{2} \alpha \beta A}{k^{\beta}} \frac{\partial \phi}{\partial k_{2}},
\end{aligned}
$$

in which we calculate from (3.1)

$$
\Lambda^{\prime} \frac{\partial \phi}{\partial k_{j}}=\bar{\sigma}_{j} k^{\beta}+\bar{A}\left(N_{j}-\alpha \bar{n}_{j}\right)>0, \quad j=1,2
$$

where $\Lambda^{\prime}>0$ is given by (3.3). We have $\partial \phi / \partial k_{j}>0, j=1,2$. The two eigenvalues, $\rho_{1}$ and $\rho_{2}$, are determined by

$$
\rho_{1,2}=\frac{a_{11}+a_{22} \pm \sqrt{\left(a_{11}-a_{22}\right)^{2}+4 a_{12} a_{21}}}{2} .
$$

It is difficult to explicitly judge the stability conditions. Simulation demonstrates that the unique equilibrium can be either stable or unstable, depending on the parameter values.

\section{Acknowledgments}

The author is grateful to important comments of Editor Huang Weihong and two anonymous referees.

\section{References}

[1] R. Solow, "A contribution to the theory of economic growth," Quarterly Journal of Economics, vol. 70, no. 1, pp. 65-94, 1956.

[2] F. Ramsey, "A mathematical theory of saving," Economic Journal, vol. 38, no. 152, pp. 543-559, 1928.

[3] P. Diamond, "National debt in a neoclassical growth model," The American Economic Review, vol. 55, pp. 1125-1150, 1965. 
[4] P. A. Samuelson, "An exact consumption-loan model of interest with or without the social contrivance of money," Journal of Political Economy, vol. 66, no. 6, pp. 467-482, 1958.

[5] E. Burmeister and A. R. Dobell, Mathematical Theories of Economic Growth, Collier Macmillan, London, UK, 1970.

[6] R. Becker and E. Burmeister, Growth Theory, vol. 3, Edward Elgar, Burlington, Vt, USA, 1991.

[7] J. B. Taylor and M. Woodford, Handbook of Macroeconomics, vol. 3, Elsevier, Amsterdam, The Netherlands, 1999.

[8] R. J. Barro and X. Sala-i-Martin, Economic Growth, McGraw-Hill, New York, NY, USA, 1995.

[9] A. B. Atkinson and F. Bourguignon, Eds., Handbook of Income Distribution, vol. 1, Elsevier, Amsterdam, The Netherlands, 2000.

[10] V. Grossmann, Inequality, Economic Growth, and Technological Change, Physica, New York, NY, USA, 2001.

[11] P. Aghion, P. Howitt, and G. L. Violante, "General purpose technology and wage inequality," Journal of Economic Growth, vol. 7, no. 4, pp. 315-345, 2002.

[12] K. Nishimura and K. Shimomura, "Trade and indeterminacy in a dynamic general equilibrium model," Journal of Economic Theory, vol. 105, no. 1, pp. 244-260, 2002.

[13] G. Sorger, "On the long-run distribution of capital in the Ramsey model," Journal of Economic Theory, vol. 105, no. 1, pp. 226-243, 2002.

[14] C. Ghiglino and G. Sorger, "Poverty traps, indeterminacy, and the wealth distribution," Journal of Economic Theory, vol. 105, no. 1, pp. 120-139, 2002.

[15] R. Becker, "On the long-run steady state in a simple dynamic model of equilibrium with heterogeneous households," Quarterly Journal of Economics, vol. 95, no. 2, pp. 375-382, 1980.

[16] O. Galor and J. Zeira, "Income distribution and macroeconomics," The Review of Economic Studies, vol. 60, no. 1, pp. 35-52, 1993.

[17] D. Acemoglu, "Technical change, inequality, and the labor market," Journal of Economic Literature, vol. 40, pp. 7-72, 2002.

[18] R. Becker and E. N. Tsyganov, "Ramsey equilibrium in a two-sector model with heterogeneous households," Journal of Economic Theory, vol. 105, no. 1, pp. 188-225, 2002.

[19] W.-B. Zhang, "A discrete economic growth model with endogenous labor," Discrete Dynamics in Nature and Society, vol. 2005, no. 2, pp. 101-109, 2005.

[20] C. Azariadis, Intertemporal Macroeconomics, Wiley-Blackwell, Oxford, UK, 1993.

[21] W.-B. Zhang, Growth with Income and Wealth Distribution, Macmillan, London, UK, 2006.

[22] A. B. Abel and B. S. Bernanke, Macroeconomics, Addison-Wesley, New York, NY, USA, 3rd edition, 1998. 


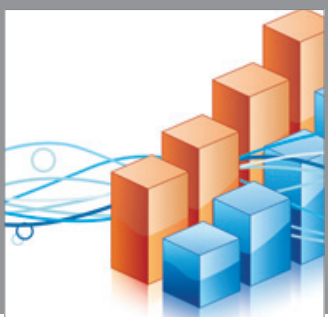

Advances in

Operations Research

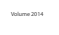

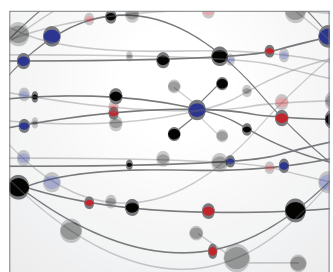

\section{The Scientific} World Journal
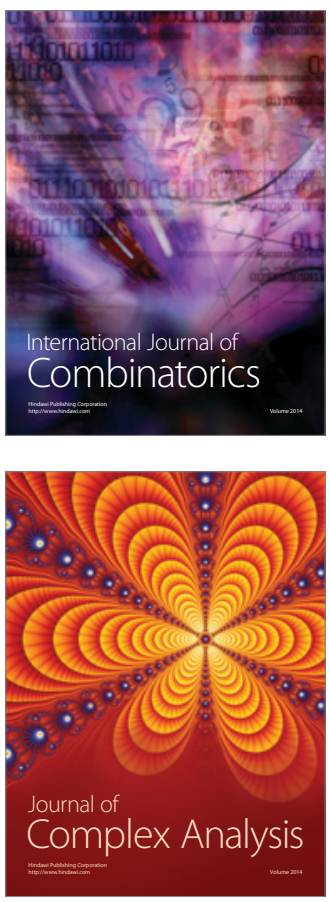

International Journal of

Mathematics and

Mathematical

Sciences
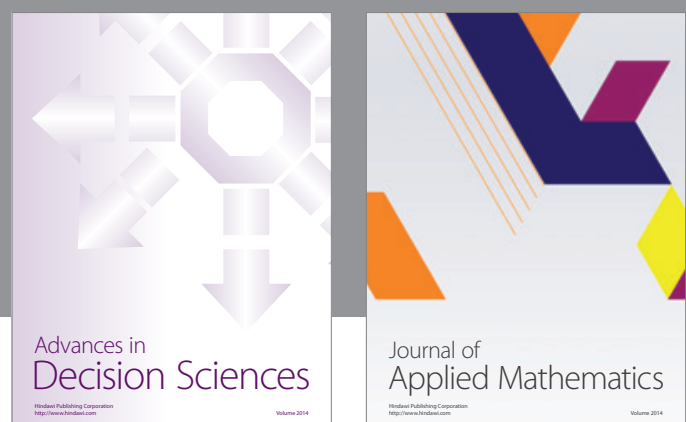

Journal of

Applied Mathematics
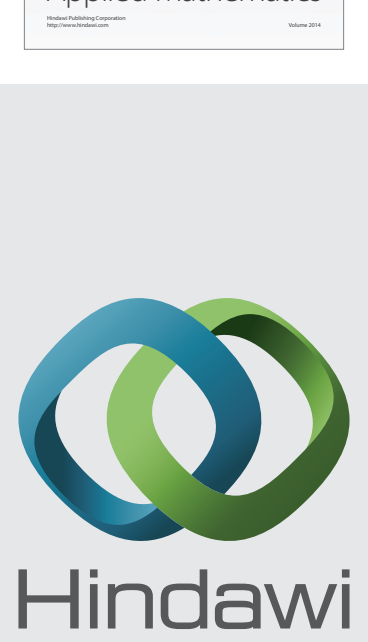

Submit your manuscripts at http://www.hindawi.com
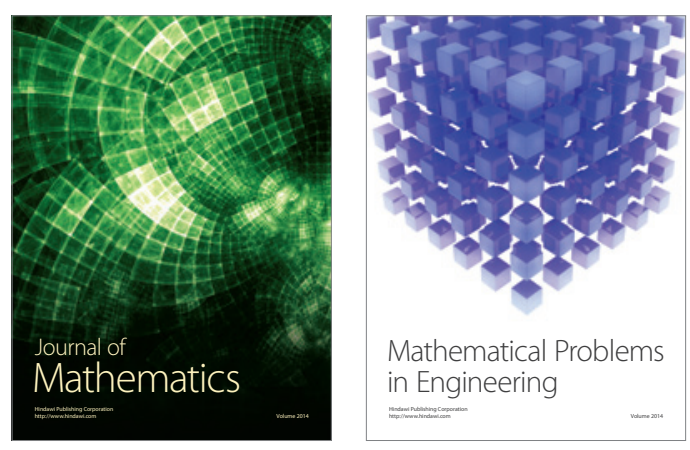

Mathematical Problems in Engineering
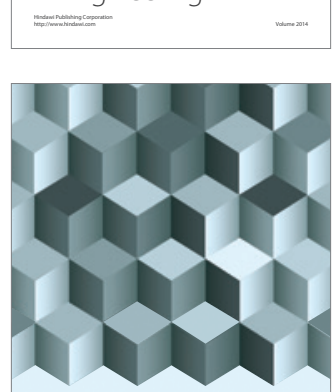

Journal of

Function Spaces
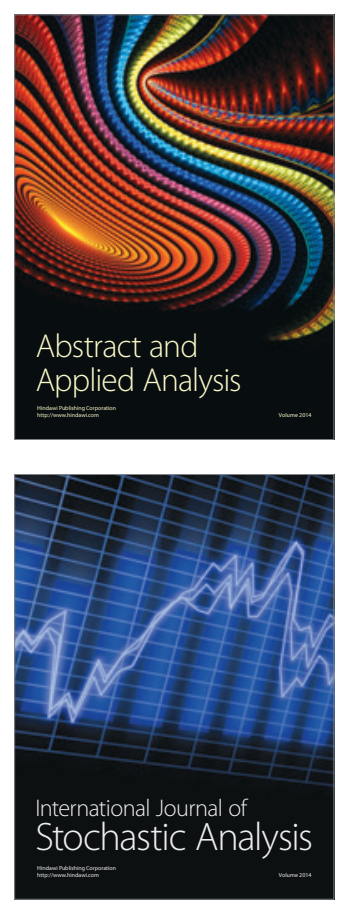

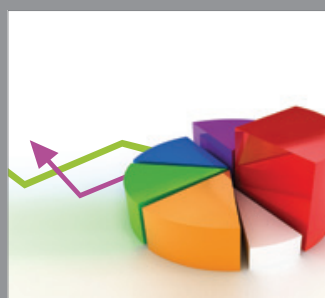

ournal of

Probability and Statistics

Promensencen
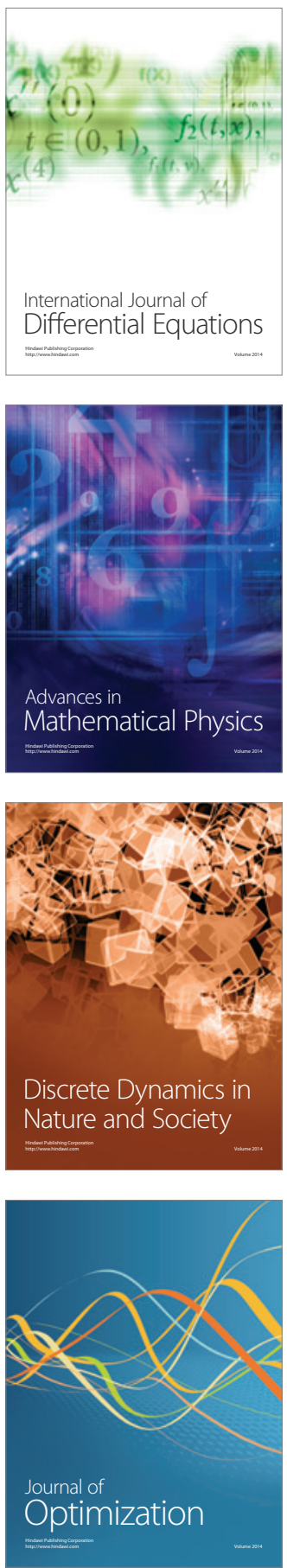